\title{
Publicly Available Rehabilitation Protocols Designated for Meniscal Repairs Are Highly Variable
}

\author{
Seth L. Carder, B.S., William G. Messamore, M.D.-Ph.D., David R. Scheffer, B.S., \\ Nick E. Giusti, B.S., John Paul Schroeppel, M.D., Scott Mullen, M.D., and \\ Bryan G. Vopat, M.D.
}

\begin{abstract}
Purpose: To compare publicly available rehabilitation protocols designated for meniscal repairs published online to determine the variability in meniscus repair protocols including different types of tears (radial vs nonradial repairs). Methods: From the Fellowship and Residency Electronic Interactive Database Access System (FREIDA), a list of publicly available academic residency programs and orthopaedic sports medicine fellowships was obtained. With this list, an electronic search using Google was performed looking for meniscal repair rehabilitation protocols. In addition to academic institutions, private practice organizations with published meniscus repair rehabilitation protocols found during the search also were examined. Results: Of 189 academic institutions, a total of 30 academic institutions had protocols that were included. Another 29 private practice programs were subsequently found and included. In total, 59 rehabilitation protocols fit the inclusion criteria. Six of the 59 specified radial repair and 53 did not. For return to full range of motion, nonradial protocols averaged 6.7 weeks and radial protocols averaged 7.3 weeks. For return to full weight-bearing, nonradial protocols averaged 6.2 weeks and radial protocols averaged 7.5 weeks. For return to sport, nonradial protocols averaged 17.8 weeks and radial protocols averaged 23.3 weeks. For time spent in a brace, nonradial protocols averaged 5.7 weeks and radial protocols averaged 6.7 weeks. Conclusions: Of publicly available meniscal repair rehabilitation protocols, a small percentage $(10.2 \%)$ changed their protocol in relation to tear type and there was a wide range of timeframes for each rehabilitation component. Protocols for radial tears tended to brace patients longer, limit their range of motion longer, delay full weight-bearing, and delay return to sport. However, it is recognized that some surgeons could be modifying their protocols in relation to tear type without publishing that information online. Clinical Relevance: As stated in the purpose, the point of this study was to access only the protocols that would be available to the public. If anything, awareness should be raised for surgeons to look at their existing protocols and update them if they are truly incomplete and outdated. More research needs to be done to structure a rehabilitation protocol that is specific to the meniscal tear type, as the current protocols have a wide range of variance.
\end{abstract}

$\mathbf{T}$ he meniscus has been studied extensively for its role in protection of the articular cartilage of the knee by distributing loads across the articular surface. ${ }^{1}$ The menisci buffer against axial, rotational, and

From the University of Kansas Medical Center, Kansas City, Kansas, U.S.A. The authors report that they have no conflicts of interest in the authorship and publication of this article. Full ICMJE author disclosure forms are available for this article online, as supplementary material.

Study was conducted at the University of Kansas Medical Center, Kansas City, Kansas, U.S.A.

Received April 28, 2020; accepted October 10, 2020.

Address correspondence to Seth Carder, B.S., 11621 E Calais Rd., Wichita, KS 67210. E-mail: Scarder@kumc.edu

(C) 2020 THE AUTHORS. Published by Elsevier Inc. on behalf of the Arthroscopy Association of North America. This is an open access article under the CC BY-NC-ND license (http://creativecommons.org/licenses/by-nc-nd/4.0/). 2666-061X/20711

https://doi.org/10.1016/j.asmr.2020.10.004 shearing forces during knee motion. ${ }^{2}$ Treatment of meniscal tears includes partial meniscectomy versus meniscal-preserving surgery. Meniscectomy has been demonstrated to increase the rate of development of osteoarthritis through finite elemental analysis, ${ }^{3}$ in vitro models, ${ }^{4}$ and long-term prospective studies. ${ }^{5-7}$ A move to meniscal preservation and repair has shown a decrease in osteoarthritis and improved outcomes particularly in younger and more active patients. $^{8-11}$

There is currently no consensus for a standardized postoperative rehabilitation protocol following meniscal repair. Within the literature, there is variation in postoperative weight-bearing, return to full range of motion (ROM), use of a brace, rate of progression through rehabilitation, and return to sport (RTS). ${ }^{12-15}$ Accelerated rehabilitation strategies with early weight-bearing have proposed benefits of decreasing the rate of 
muscle atrophy and strength deficits, ${ }^{16}$ and faster RTS without an increase in complications, ${ }^{17}$ although these studies rarely stratified meniscal repairs based on tear patterns or characteristics.

Postoperative protocols intending to decrease the risk of retear following repair may restrict the patient's ROM. Cadaveric studies have shown that femorotibial contact pressure increases with knee flexion. ${ }^{18} \mathrm{Re}$ striction of ROM may protect a meniscal repair from increased mechanical stress. ${ }^{19}$ In addition, the effect of postoperative weight-bearing on the force across a meniscal repair varies based on the tear pattern and location. $^{20}$ Vertical longitudinal tears experience compressive forces at the repair site with loading and early weight-bearing may help to reduce the tear and aid in healing, whereas radial tears experience distraction forces and increased strain with weight-bearing. ${ }^{20}$ Because of biomechanical factors, postoperative ROM and weight-bearing status can impact healing after repair, the interplay between tear type and knee biomechanics should guide postoperative plans. ${ }^{13}$ The purpose is to compare publicly available rehabilitation protocols designated for meniscal repairs published online to determine the variability in radial versus nonradial repair protocols including different types of tears (radial vs nonradial repair). We hypothesized that there would be a high variability in postoperative rehabilitation protocols.

\section{Methods}

From the Fellowship and Residency Electronic Interactive Database Access System (FREIDA), a comprehensive list of publicly available academic residency programs and orthopaedic sports medicine fellowships was obtained. With this list, an electronic search using Google's (www.google.com) search engine was performed using the following search phrase, "[Program/ affiliate hospital/affiliate medical school name] meniscus repair rehabilitation protocol." In addition to academic institutions, private practice organizations with published meniscus repair rehabilitation protocols that appeared during the electronic search also were examined to determine whether they fit the inclusion criteria. References were limited to the first page of search results.

Protocols were included if written in the English language and specified rehabilitation following meniscal repairs. Included protocols were further separated into groups if they indicated rehabilitation for a specific type of meniscal repair (peripheral, root, radial, etc.). Protocols were excluded if not written in the English language, included other surgical repairs besides meniscus, and/or did not include time-based components specifying expected time to full ROM and time to full weight-bearing status. Studies were still included if they did not report time in a brace and RTS if the previous requirements were met.

The following rehabilitation components were used to compare the rehabilitation protocols: ROM, time in a brace, time until initiation of weight-bearing, and RTS. The primary outcome of this study was the timing of each rehabilitation component, as well as the incidence, inclusion, or exclusion, of the aforementioned components while secondarily examining whether there were separate protocols for radial and nonradial meniscus tears

When we compared the time-based parameters, the initial number was used if the protocol specified a range of time. For example, if the protocol specified return to full ROM by 4 to 6 weeks, 4 weeks was included as the data point. While many protocols recommended immediate active ROM of the hip and ankle, this study only assessed recommendations for knee ROM.

\section{Results}

A flow chart of protocol selection can be found in Fig 1. Of the 189 academic institutions included in the FREIDA online residency program database, a total of 30 academic institutions had publicly available rehabilitation protocols designated for meniscal tears. An additional 29 private practice programs were subsequently found during the FREIDA search that also had publicly available rehabilitation protocols designated for meniscal tears. In total, 59 rehabilitation protocols were deemed to fit the inclusion criteria. Of these 59 protocols, $53(89.8 \%)$ did not include specific instructions for radial meniscus tears and 6 protocols $(10.2 \%)$ included specific instructions for radial meniscus tears. From the 53 protocols that did not specify a radial meniscus tear, $26(49.1 \%)$ were from an academic institution and $27(50.9 \%)$ were private practice. Of the 6 that did specify radial meniscus tears, $4(66.7 \%)$ were from academic institutions and $2(33.3 \%)$ came from private practices.

\section{Range of Motion}

The timeline to return to full ROM from all the protocols were compared. All $59(100 \%)$ included protocols reported ROM. The time to return to full ROM for nonradial meniscus protocols $(n=53)$ had the following breakdown: $7(13.2 \%)$ reported return to ROM at 4 weeks, $4(7.5 \%)$ reported return to ROM at 5 weeks, $24(45.3 \%)$ reported return to ROM at 6 weeks, $1(1.9 \%)$ reported return to ROM at 7 weeks, 11 $(20.7 \%)$ reported return to ROM at 8 weeks, $3(5.7 \%)$ reported return to ROM at 10 weeks, and $3(5.7 \%)$ reported return to ROM at 12 weeks (Fig 2A). Most of these protocols $(n=24)$ indicated that full ROM should be achieved at 6 weeks. This resulted in a cumulative average of 6.7 weeks between repair and full ROM following nonradial meniscus repair. 
Fig 1. Flow diagram used for the study. (FRIEDA, Fellowship and Residency Electronic 4 Interactive Database Access System; Radial and Non-Radial meniscus tear.)

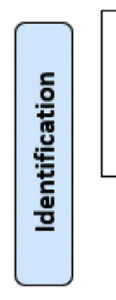

Academic Institutions identified per FREIDA online residency program database ( $n=189$ )
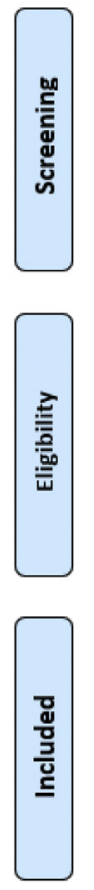
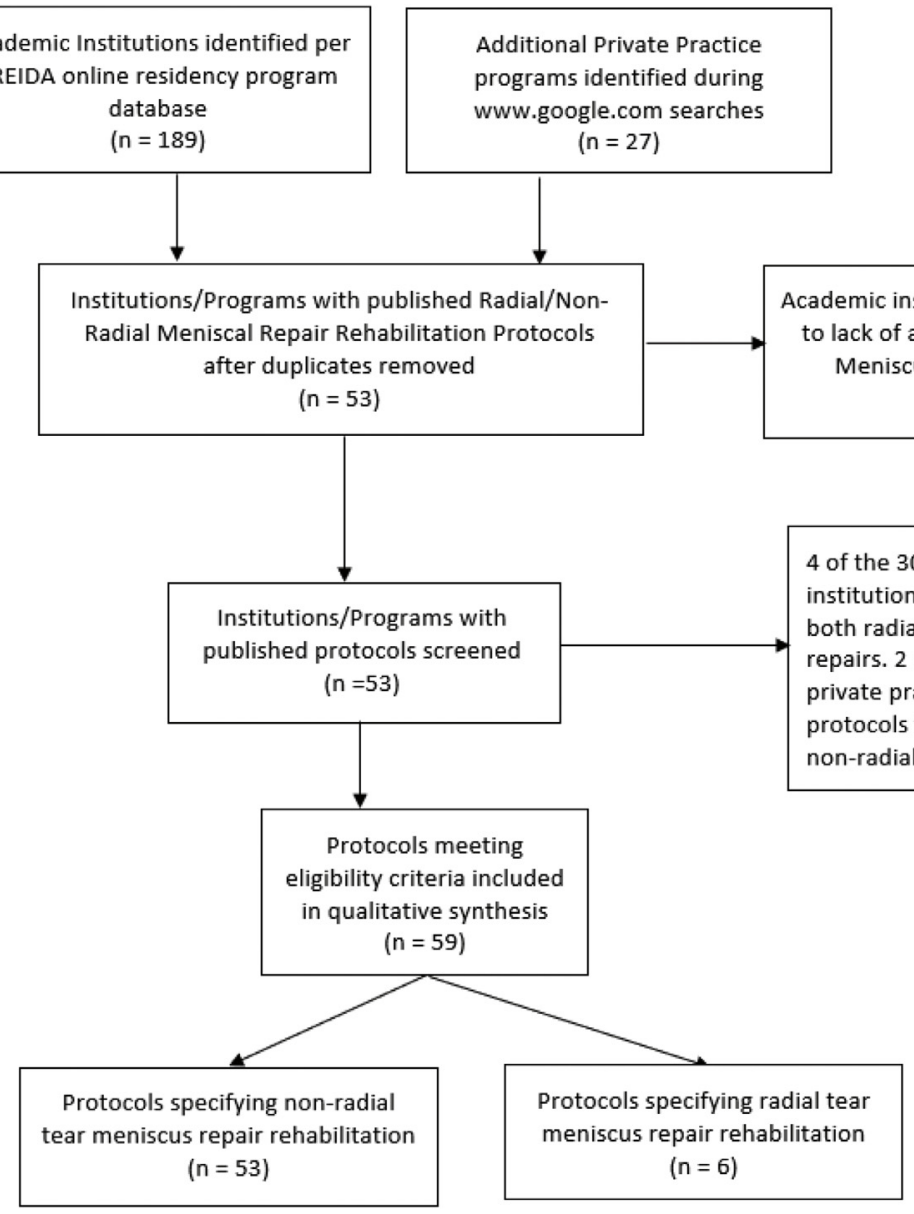

All $6(100 \%)$ of the included radial protocols included a time to return to full ROM. The timelines for full ROM following radial meniscus repairs included: $1(16.7 \%)$ reported return to ROM at 6 weeks, $2(33.3 \%)$ reported return to ROM at 7 weeks, and $3(50.0 \%)$ reported return to ROM at 8 weeks (Fig 2B). While the distribution of data for the radial meniscus protocols was more compact, the majority $(n=3)$ indicated that full ROM be achieved by 8 weeks. This resulted in a cumulative average of 7.3 weeks between repair and full ROM following radial meniscus repair.

Comparison between number of protocols as well as the recommended week to return to full ROM in radial and non-radial meniscus repairs can be seen in Fig 2C.

\section{Return to Full Weight-Bearing (FWB)}

The timeline to return to FWB from all the protocols were compared. All 59 (100\%) included protocols reported FWB. The time to return to FWB for nonradial meniscus protocols $(n=53)$ had the following breakdown: $1(1.9 \%)$ reported return to FWB at 2 weeks, 3 $(3.8 \%)$ reported return to FWB at 3 weeks, 13 (24.5\%) reported return to FWB at 4 weeks, $3(5.7 \%)$ reported return to FWB at 5 weeks, $22(41.5 \%)$ reported return to FWB at 6 weeks, $6(11.3 \%)$ reported return to FWB at 8 weeks, $1(1.9 \%)$ reported return to FWB at 11 weeks, and $5(9.4 \%)$ reported return to FWB at 12 weeks (Fig 3A). The majority of these protocols $(\mathrm{n}=$ 22 ) indicated that FWB should be achieved at 6 weeks. This resulted in a cumulative average of 6.2 weeks between repair and RTS following nonradial meniscus repair.

All $6(100 \%)$ of the included radial protocols included a time to return to FWB. The timelines for FWB following radial meniscus repairs included: $3(50.0 \%)$ reported return to FWB at 7 weeks and $3(50.0 \%)$ reported return to FWB at 8 weeks (Fig 3B). These data are more concise and agrees that FWB status after radial meniscus repair should be obtained by weeks 7 or 8 . This resulted in a cumulative average of 7.5 weeks between repair and FWB status following radial meniscus repair.

Comparison between number of protocols as well as the recommended week to return to FWB in radial and nonradial meniscus repairs can be seen in Fig 3C. 

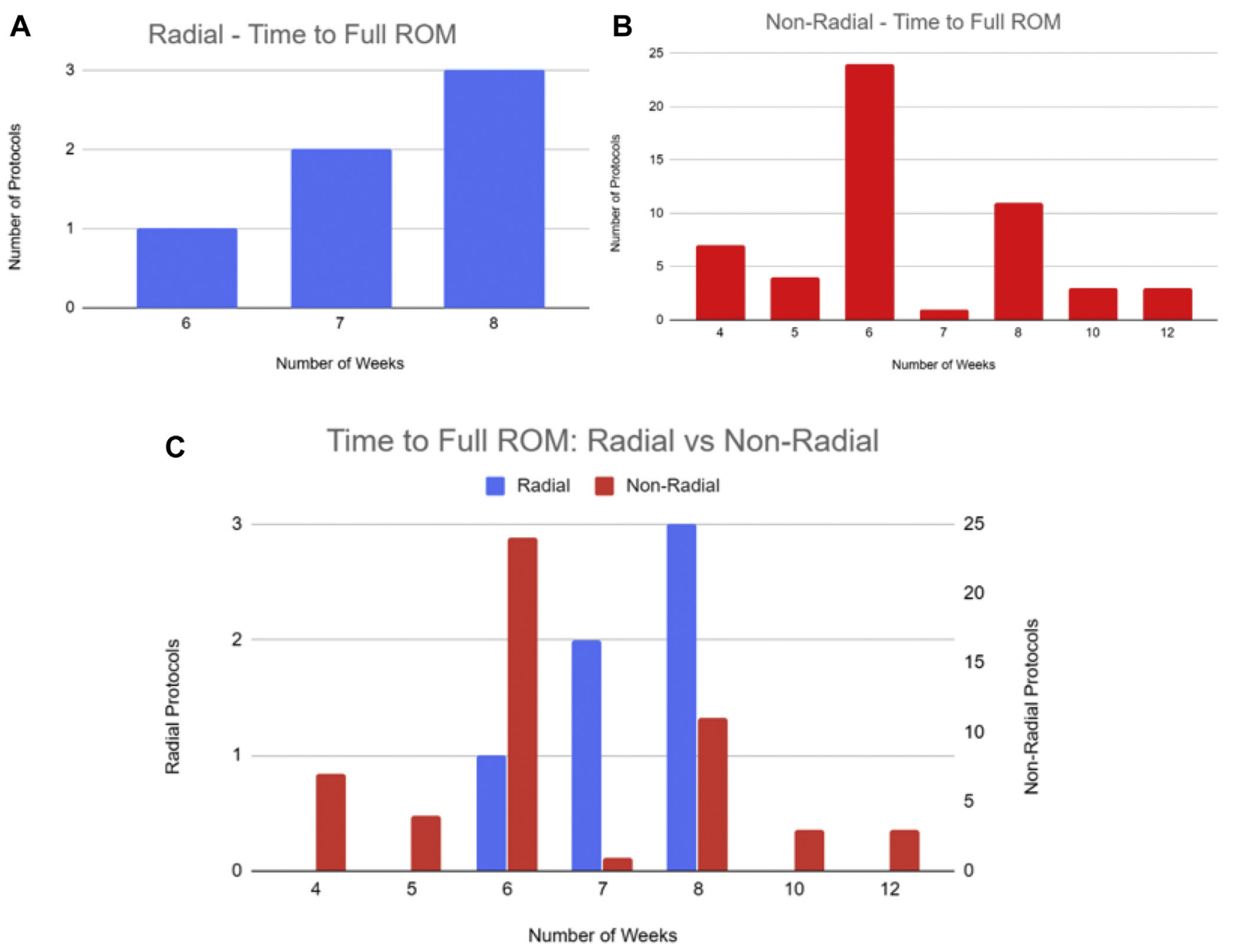

Fig 2. (A-C) Recommendations for return to full ROM following radial meniscus repair (A), nonradial meniscus repair (B), and the comparison between radial and nonradial return to full ROM (C). (ROM, range of motion.)

\section{Return to Sport}

The timeline for RTS was compared between the included protocols. For nonradial meniscus protocols $(\mathrm{n}=53)$, there were 50 protocols $(94.3 \%)$ which provided a timeline for RTS, 3 protocols $(5.6 \%)$ did not provide a timeline. The breakdown for studies reporting a RTS timeline for radial meniscus repairs included: 9 studies $(17.0 \%)$ reported RTS in 12 weeks, 23 studies $(43.4 \%)$ reported RTS in 16 weeks, 9 studies $(17.0 \%)$ reported RTS in 20 weeks, 1 study ( $1.9 \%)$ reported RTS in 22 weeks, 5 studies $(9.4 \%)$ reported RTS in 24 weeks, 2 studies $(3.8 \%)$ reported RTS in 28 weeks, and l study $(1.9 \%)$ reported RTS in 36 weeks. The majority of the protocols $(n=23)$ reported RTS in 16 weeks following nonradial meniscus repair as seen in Fig 4A. This resulted in a cumulative average of 17.8 weeks between repair and RTS following nonradial meniscus repair.

For radial meniscus protocols $(\mathrm{n}=6)$, all 6 provided a timeline for RTS $(100 \%)$. The timelines for RTS following radial meniscus repairs included $2(33.3 \%)$ studies reported RTS in 16 weeks, 3 studies $(50 \%)$ reported RTS in 24 weeks, and 1 study $(16.7 \%)$ reported RTS in 36 weeks. The majority of the protocols $(n=3)$ reported RTS in 24 weeks following radial meniscus repair as seen in Fig $4 \mathrm{~B}$. This resulted in a cumulative average of 23.3 weeks between repair and RTS following radial meniscus repair.

Comparison between number of protocols as well as the recommended week to RTS in radial and nonradial meniscus repairs can be seen in Fig 4C.

\section{Time in Brace (TI)}

The timeline for time in a brace was compared between the included protocols. For nonradial meniscus protocols $(n=53)$, there were 51 protocols $(96.2 \%)$ which provided a timeline for TI, and 2 protocols $(3.8 \%)$ did not provide a timeline. The breakdown for studies reporting a TI timeline for radial meniscus repairs included 1 study $(1.9 \%)$ reported TI of 3 


\section{A}

Radial - Time to Full Weight Bearing 6

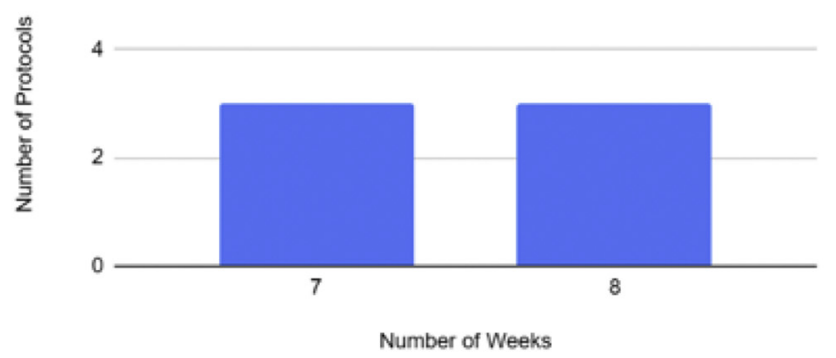

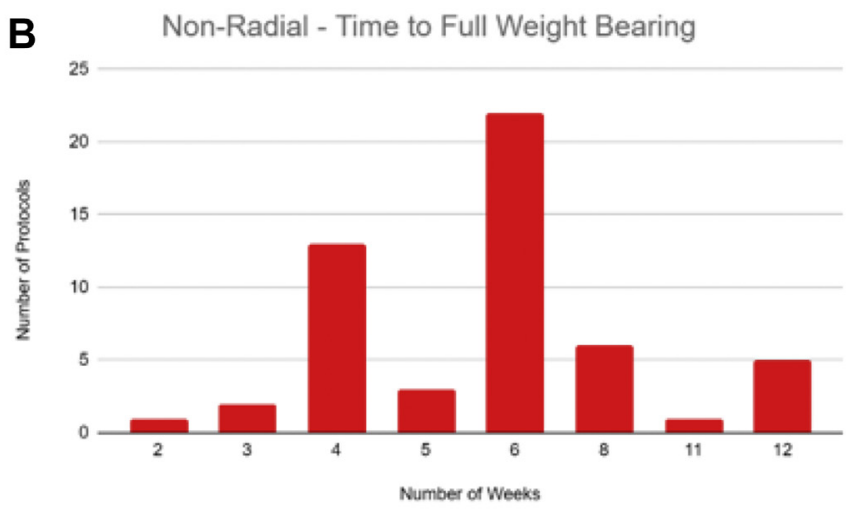

\section{Time to Full Weight Bearing: Radial vs Non-Radial \\ Radial $\square$ Non-Radial}

6 25

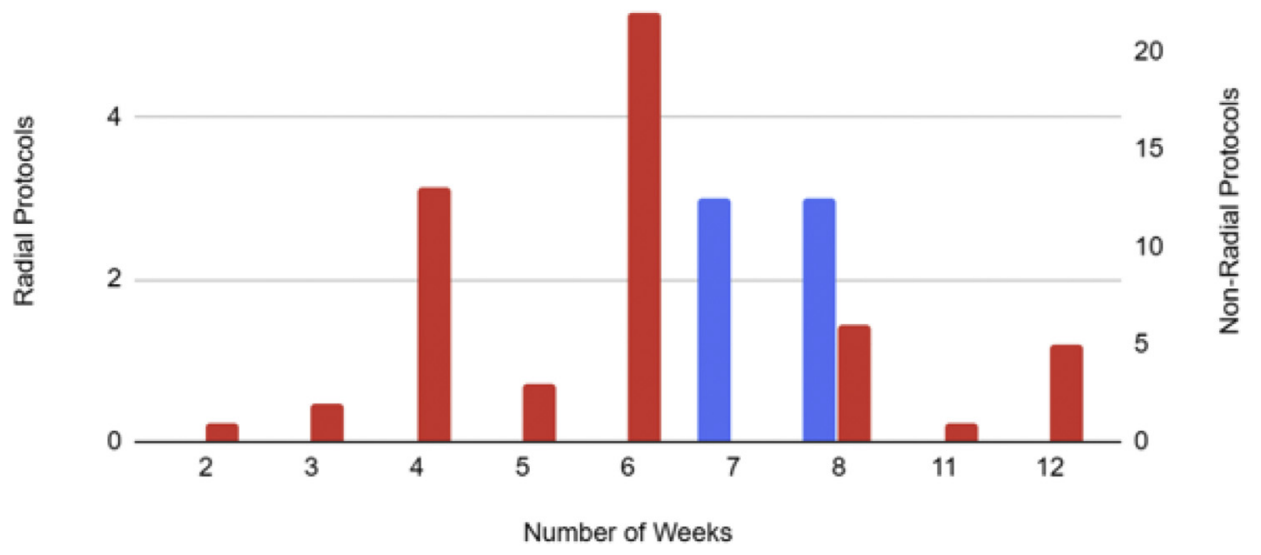

Fig 3. (A-C) Recommendations for return to full weight-bearing following radial meniscus repair (A), nonradial meniscus repair (B), and the comparison between radial and nonradial return to full weight-bearing (C).

weeks, 11 studies $(20.8 \%)$ reported TI of 4 weeks, 1 study $(1.9 \%)$ reported TI of 5 weeks, 33 studies $(62.3 \%)$ reported TI of 6 weeks, 4 studies $(7.5 \%)$ reported TI of 8 weeks, and 1 study $(1.9 \%)$ reported TI of 10 weeks. The majority of the protocols $(n=33)$ reported TI of 6 weeks following nonradial meniscus repair, as seen in Fig 5A. This resulted in a cumulative average of 5.7 weeks of bracing following nonradial meniscus repair.

For radial meniscus protocols $(\mathrm{n}=6)$, all 6 provided a timeline for TI (100\%). The timelines for TI following radial meniscus repairs included 1 study $(16.7 \%)$ reported TI of 4 weeks, 1 study $(16.7 \%)$ reported TI of 6 weeks, 2 studies $(33.3 \%)$ reported TI of 7 weeks, and 2 studies $(33.3 \%)$ reported TI of 8 weeks. There was no majority between protocols as a TI of 7 weeks and TI of 8 weeks was reported in 2 protocols each as seen in Fig 5B. This resulted in a cumulative average of 6.7 weeks of bracing following radial meniscus repair.
Comparison between number of protocols as well as the recommended week to discontinue bracing in radial and nonradial meniscus repairs can be seen in Fig 5C.

\section{Discussion}

This study shows that there is a lack of consensus between the various publicly available rehabilitation protocols after meniscus repair. Despite the effect of postoperative weight-bearing and ROM on the force across a meniscal repair varying based on the tear pattern and location, we found that most institutions $(89.8 \%)$ have not published publicly available separate guidelines for rehabilitation based on tear pattern or location. There was also heterogeneity between published rehabilitation protocols with regard to initiation of weight-bearing, length of bracing, time to full ROM, and RTS.

With respect to studies looking at accelerated and traditional rehabilitation following meniscal repair, there are a lack of data demonstrating the advantage of 

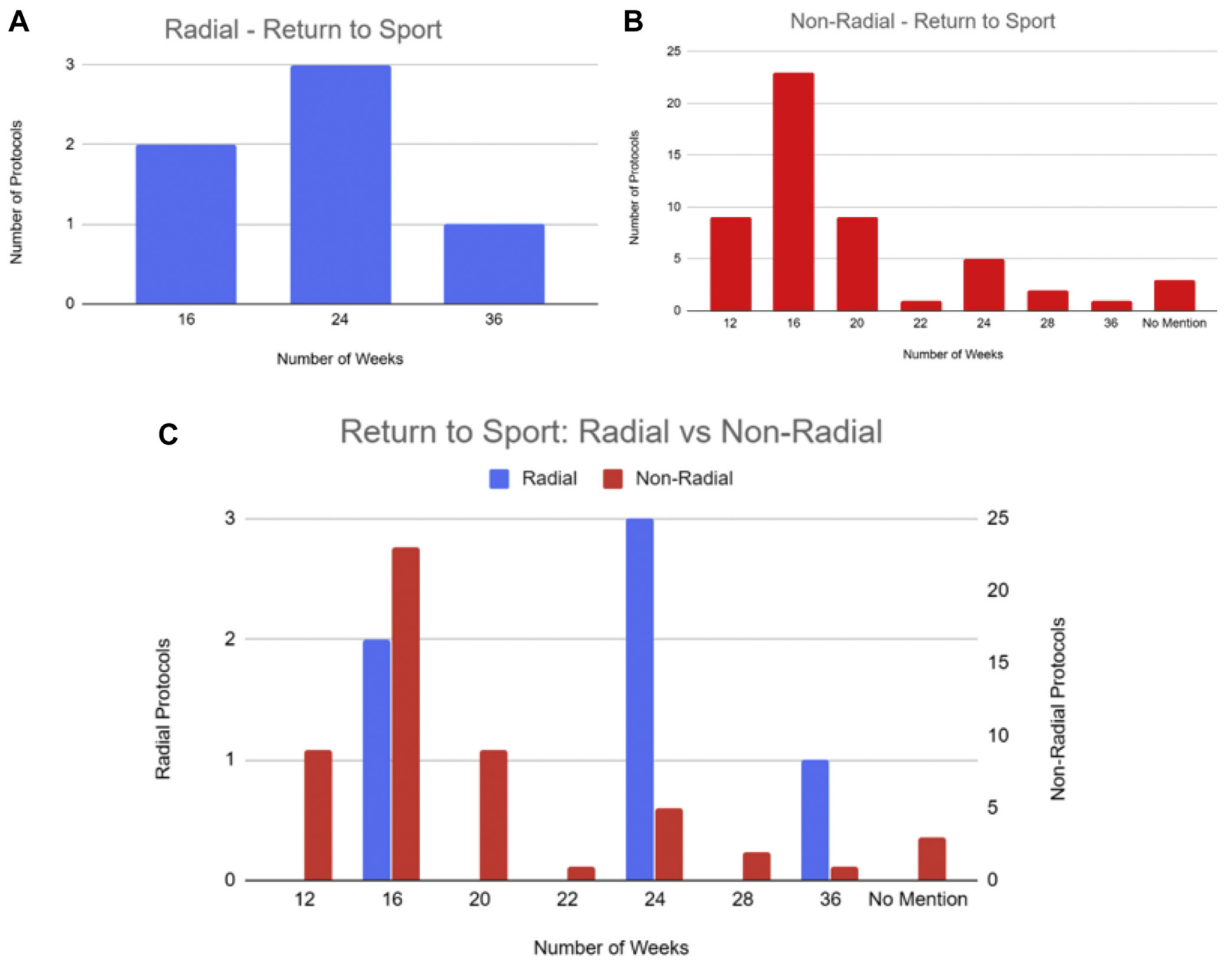

Fig 4. (A-C) Recommendations for return to sport following radial meniscus repair (A), nonradial meniscus repair (B), and the comparison between radial and nonradial full weight-bearing $(\mathrm{C})$.

accelerated rehabilitation for specific tear patterns. A study by Mariani et al. ${ }^{18}$ was done that put subject through an accelerated protocol only. All their subjects underwent a meniscal repair using the outside-in technique and also anterior cruciate ligament reconstruction. Outcome criteria were assessed via clinical outcome and magnetic resonance imaging. Their results demonstrate meniscal retear in only $13.6 \%$ of subjects undergoing the accelerated rehabilitation protocol. This study had 2 limitations: inclusion of anterior cruciate ligament reconstructions and, more concerning, a lack of a control group to make a comparison with. Thus, the results cannot be extrapolated to fit all meniscal rehabilitation protocols. ${ }^{18}$

Barber ${ }^{19}$ published a study that compared the traditional approach to rehabilitation to the accelerated approach. The traditional group had 58 subjects with an average age of 23 years and had a failure rate of $19 \%$. The accelerated group had 40 subjects with an average age of 26 years and a failure rate of $10 \%$. Therefore, the authors concluded that there was no statistical difference between the 2 groups and that there was no need to activity restrictions after meniscus repair. This study was limited by its average age of subject and also that most of the subjects were athletes trying to return to sports. Another major limitation to this study is that they authors did not specify meniscus tear types. ${ }^{19}$

A randomized control trial published in 2013 (level 1 evidence) examined the relationship between traditional and accelerated meniscal repair protocols. The accelerated group was never braced and only had 2 weeks' worth of restrictions on ROM and weightbearing. The traditional group was put in a brace for 6 weeks and had gradual return to ROM and weightbearing over the 6 weeks. The patients were then followed for 2 years to track outcomes. The authors found no significant increase in failure rates in the accelerated group over the traditional group. There was also no 
A

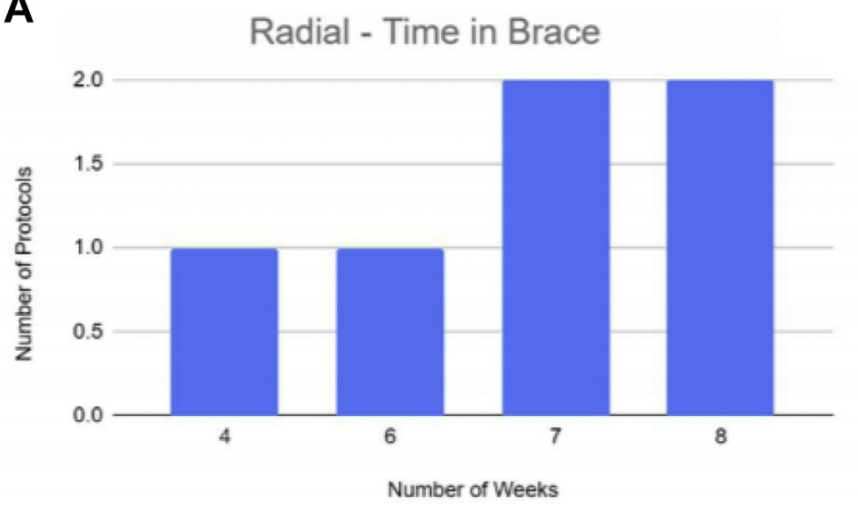

B

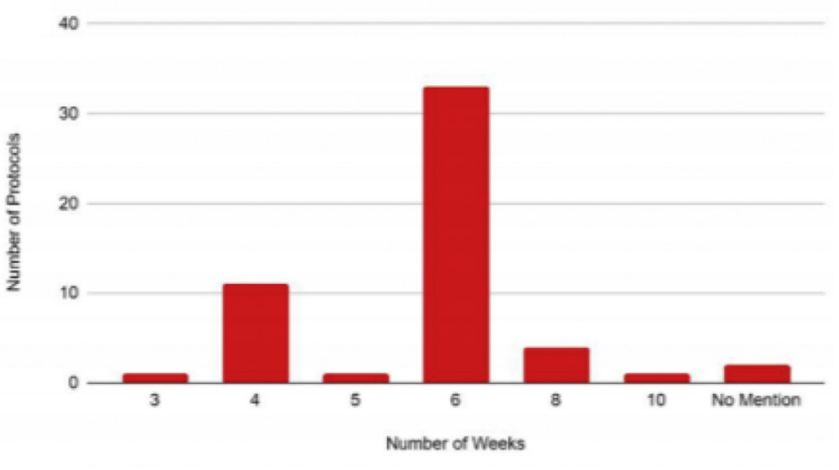

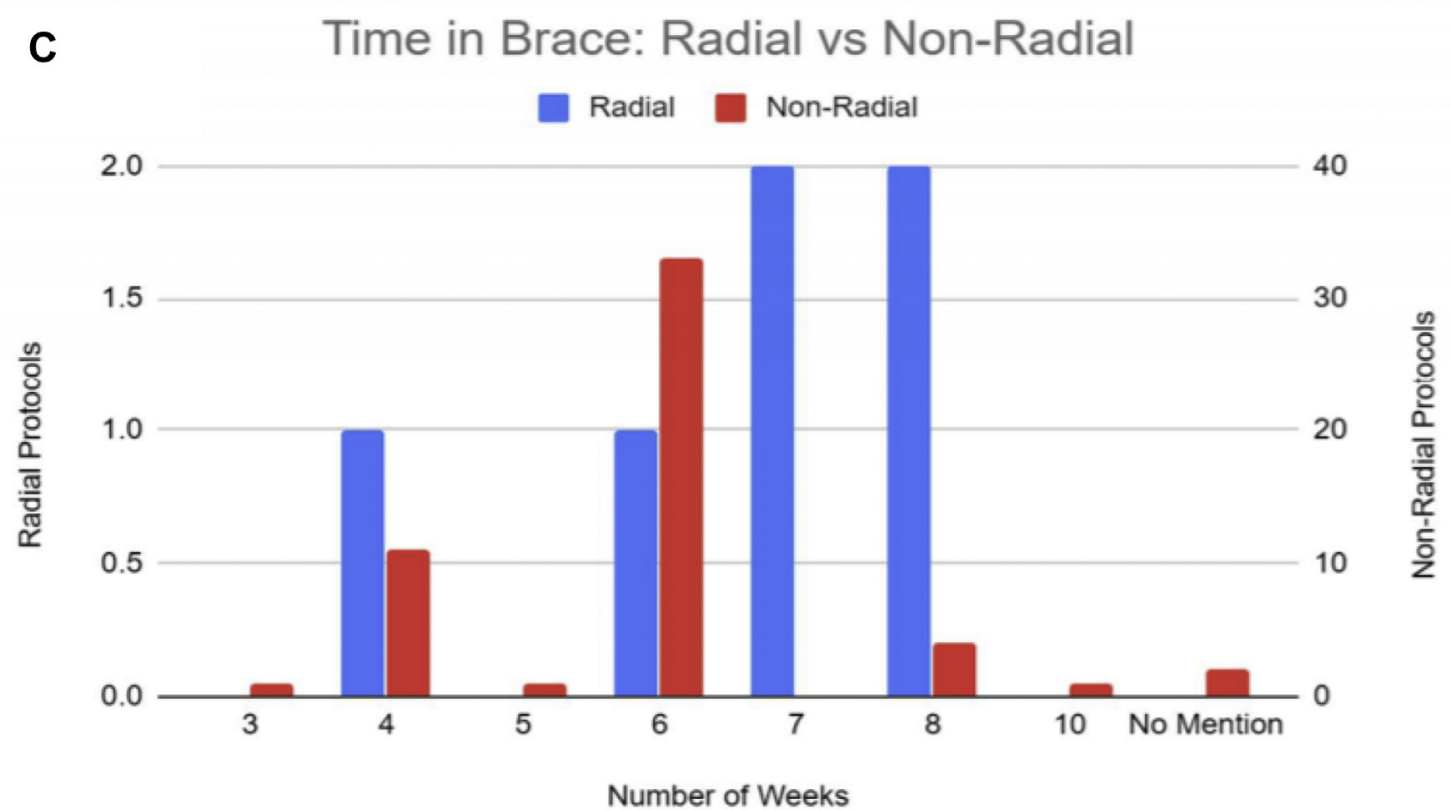

Fig 5. (A-C) Recommendations for time spent in a brace following radial meniscus repair (A), nonradial meniscus repair (B), and the comparison between radial and nonradial time in a brace $(\mathrm{C})$.

difference in functional outcomes. However, this study was limited by enrolling subjects who only had vertically oriented meniscal tears. While some cite this as evidence that accelerated protocols work, it needs to be noted that there is a noticeable lack of data suggesting the accelerated approach is safe in radial and root meniscal tears. ${ }^{20}$

We believe it is important individualize meniscal rehabilitation protocols to not just the tear pattern, but also to the functional goals of the patient. Cavanaugh et al. ${ }^{15}$ noted that clinical guidelines developed by treating clinicians and the referring surgeons should incorporate flexible time frames in the progression to allow for individualization, for example: "week 4 to 6 -discontinue crutches when non-antalgic gait is demonstrated" versus "week 6-discontinue crutches for ambulation." Continual reassessment of the patient is vital to ensure a consistent and safe progression of the program. Therapeutic exercise programs, therefore, often must be modified based on changes in subjective and objective findings." ${ }^{15}$ The authors of this paper agree with this statement from Cavanaugh et al. Flexible guidelines with individually targeted goals should be developed to help appropriately guide the rehabilitation process.

Despite studies showing that increased knee flexion increases the femorotibial contact pressure and that restriction of motion may protect certain repair types from increased mechanical stress there was a wide range in return to full ROM in the rehabilitation protocols studied. ${ }^{21,22}$ Some protocols $(13.2 \%)$ returned patients to full ROM as early as 4 weeks, whereas others $(5.7 \%)$ delayed full ROM for a full 12 weeks. Protocols that specified radial tears were more likely to delay return to full ROM longer than other tear types, 7.3 weeks on average versus 6.7 weeks on average. In addition, the recommended time in a brace was longer for protocols that specified radial tears (6.7 weeks' 
average) than those that did not (5.7 weeks' on average).

As previously mentioned, certain meniscal tear patterns may benefit from early return to weight-bearing whereas weight-bearing may put repairs at risk for radial tears. ${ }^{23}$ Recommended time until initiation of weight-bearing varied from 2 weeks to 12 weeks in protocols that did not distinguish between tear type. Radial tear specific protocols recommended a more uniform 7 to 8 weeks return to full weight-bearing and were more likely to delay weight-bearing longer, 7.5 weeks on average versus 6.2 weeks on average.

RTS as an outcome is highly variable based off its definition (return to participation, return to a specific sport, or return to performance), sport type (highimpact vs low-impact), and sport level (professional vs recreational). As such it is an ill-defined parameter, but at the minimum usually involves pain-free return to full ROM and with impact activities including running and/or plyometric exercise. Again, we see heterogeneity amongst the protocols studied from 12 to 36 weeks, and again a delay in RTS with radial tear-specific protocols with RTS at 23.3 weeks on average versus 17.8 weeks in the other protocols.

\section{Limitations}

There are certain limitations to this study that should be taken into consideration. While there were 59 protocols included in this study, there are likely additional rehabilitation protocols that would have fit the inclusion criteria but were unable to be included because they have not been published online for public access. Furthermore, there are likely additional rehabilitation protocols published by private practice organizations that were not discovered during the electronic search. This is mainly because the Google search only included the names of academic institutions but not private practice organizations. Without a comprehensive list of national private practices organizations in the field of orthopaedics and sports medicine, an all-inclusive search with academic institutions and private practice organizations could not be completed without generating selection bias.

Another limitation would be that radial tears can be divided into root and non-root varieties. Both of these injuries experience distraction forces with weightbearing, but due to the limited variety of tear-specific protocols found, we did not look specifically at the differences in radial and root tears.

\section{Conclusions}

Of publicly available meniscal repair rehabilitation protocols, a small percentage $(10.2 \%)$ changed their protocol in relation to tear type, and there was a wide range of time frames for each rehabilitation component.
Protocols for radial tears tended to brace patients longer, limit their ROM longer, delay full weightbearing, and delay RTS. However, it is recognized that some surgeons could be modifying their protocols in relation to tear type without publishing that information online.

\section{References}

1. Baratz ME, Fu FH, Mengato R. Meniscal tears: The effect of meniscectomy and of repair on intraarticular contact areas and stress in the human knee. Am J Sports Med 1986;14:270-275.

2. Kurosawa H, Fukubayashi T, Nakajima H. Load-bearing mode of the knee joint: Physical behavior of the knee joint with or without menisci. Clin Orthop Relat Res 1980;149: 283-290.

3. Dong Y, Hu G, Dong Y, Hu Y, Xu Q. The effect of meniscal tears and resultant partial meniscectomies on the knee contact stresses: A finite element analysis. Comput Methods Biomech Biomed Engin 2014;17:1452-1463.

4. Muriuki MG, Tuason DA, Tucker BG, Harner CD. Changes in tibiofemoral contact mechanics following radial split and vertical tears of the medial meniscus: An in vitro investigation of the efficacy of arthroscopic repair. J Bone Joint Surg Am 201 1;93:1089-1095.

5. Pengas IP, Assiotis A, Nash W, Hatcher J, Banks J, McNicholas MJ. Total meniscectomy in adolescents: A 40year follow-up. J Bone Joint Surg Br 2012;94B:1649-1654.

6. Roos H, Laurén M, Adalberth T, Roos E, Jonsson K, Lohmander LS. Knee osteoarthritis after meniscectomy. Arthritis Rheum 2005:41:687-693.

7. Roos EM, Östenberg A, Roos H, Ekdahl C, Lohmander LS. Long-term outcome of meniscectomy: Symptoms, function, and performance tests in patients with or without radiographic osteoarthritis compared to matched controls. Osteoarthr Cartil 2001;9:316-324.

8. Petty CA, Lubowitz JH. Does arthroscopic partial meniscectomy result in knee osteoarthritis? A systematic review with a minimum of 8 years' follow-up. Arthroscopy 2011;27:419-424.

9. Englund M, Roos EM, Roos HP, Lohmander LS. Patientrelevant outcomes fourteen years after meniscectomy: Influence of type of meniscal tear and size of resection. Rheumatology 2001;40:631-639.

10. Seitz AM, Lubomierski A, Friemert B, Ignatius A, Dürselen L. Effect of partial meniscectomy at the medial posterior horn on tibiofemoral contact mechanics and meniscal hoop strains in human knees. J Orthop Res 2012;30:934-942.

11. Stein T, Mehling AP, Welsch F, von Eisenhart-Rothe R, Jäger A. Long-term outcome after arthroscopic meniscal repair versus arthroscopic partial meniscectomy for traumatic meniscal tears. Am J Sports Med 2010;38:1542-1548.

12. O'Donnell K, Freedman KB, Tjoumakaris FP. Rehabilitation protocols after isolated meniscal repair: A systematic review. Am J Sports Med 2017;45:1687-1697.

13. Spang RC III, Nasr MC, Mohamadi A, DeAngelis JP, Nazarian A, Ramappa AJ. Rehabilitation following meniscal repair: A systematic review. BMJ Open Sport Exerc Med 2018;4:e000212. 
14. VanderHave KL, Perkins C, Le M. Weightbearing versus nonweightbearing after meniscus repair. Sports Health 2015;7:399-402.

15. Cavanaugh JT, Killian SE. Rehabilitation following meniscal repair. Curr Rev Musculoskelet Med 2012;5: 46-58.

16. Wright RW, Fetzer GB. Bracing after ACL reconstruction: A systematic review. Clin Orthop Relat Res 2007;455: 162-168.

17. Shelbourne KD, Patel DV, Adsit WS, Porter DA. Rehabilitation after meniscal repair. Clin Sports Med 1996;15:595-612.

18. Mariani PP, Santori N, Adriani E, Mastantuono M. Accelerated rehabilitation after arthroscopic meniscal repair: A clinical and magnetic resonance imaging evaluation. Arthroscopy 1996;12:680-686.

19. Barber FA. Accelerated rehabilitation for meniscus repairs. Arthroscopy 1994;10:206-210.
20. Lind M, Nielsen T, Faunø P, Lund B, Christiansen SE. Free rehabilitation is safe after isolated meniscus repair: A prospective randomized trial comparing free with restricted rehabilitation regimens. Am J Sports Med 2013;41:2753-2758.

21. Becker R, Wirz D, Wolf C, Göpfert B, Nebelung W, Friederich N. Measurement of meniscofemoral contact pressure after repair of bucket-handle tears with biodegradable implants. Arch Orthop Trauma Surg 2005;125: 254-260.

22. Anderson DR, Gershuni DH, Nakhostine M, Danzig LA. The effects of non-weight-bearing and limited motion on the tensile properties of the meniscus. Arthroscopy 1993;9: 440-445.

23. Jones RS, Keene G, Learmonth D, et al. Direct measurement of hoop strains in the intact and torn human medial meniscus. Clin Biomech 1996;1 1:295-300. 